\title{
A Review on Molecular Marker Analysis for Yield and its Component Traits under Water Stress and Zinc Deficiency Tolerance in Rice
}

\author{
J. Vanitha*, K. Amudha, R. Mahendran, J. Srinivasan and R. Usha Kumari \\ Tamilnadu Agricultural University, Coimbatore, Tamil Nadu 641003, India \\ *Corresponding author
}

\section{A B S T R A C T}

\section{Keywords}

Aerobic rice, Molecular marker analysis, Zinc deficiency tolerance and Yield and its component traits

Article Info

Accepted:

10 April 2019

Available Online:

10 May 2019
The Wonder Cereal, Rice (Oryza sativa L.) is the heart of our culture and the staple food crop consumed by more than 50 per cent of the world's population. Aerobic rice proves to be a viable technology by reducing water losses through seepage, percolation and evaporation. However, under aerobic condition several essential nutrients, especially zinc became unavailable due to positive soil redox potential. Therefore genetic improvement of rice genotypes for zinc deficiency under aerobic condition is essential to exploit the water saving potential of aerobic condition. Molecular markers augment conventional plant breeding for efficient and precise identification or selection of a trait of interest linked to them.

\section{Introduction}

During the last few decades, molecular markers have been immensely used in plant biotechnology and their genetics studies. Microsatellites are tandem repeats of DNA sequences of only a few base pairs (1 - 6 bp) in length, the most abundant being dinucleotide repeats (Morgante and Olivievi, 1993). The completion of rice genome sequence provided an opportunity to identify thousands of new targets for DNA markers, especially SSRs. There were 18,828 SSRs (di, tri-, tetra-repeats) released after the completion of the Nipponbare genome sequence in 2005. It is estimated that, the density of SSRs (approx. 51 SSRs per $\mathrm{Mb}$ ) can provide a considerable map construction and MAS for numerous applications.

\section{Zinc transporters}

Zn transporters play a central role in plant acquisition of zinc from soil and its distribution. Many different $\mathrm{Zn}$ transporters have been identified and they are distributed throughout the plant system. The maintenance of $\mathrm{Zn}$ homeostasis in whole plant relies on a variety of transporters, including the members of zinc-regulated transporter (ZRT) and iron regulated transporter (IRT) like protein $(Z I P)$ which are involved in the cellular uptake of 
$\mathrm{Zn}$ from soil to root cells at soil root interface (Colangelo and Guerinot, 2006); natural resistance associated macrophage protein (NRAMP) families which regulate the proton driven transport of $\mathrm{Zn}$ and other transition metal ions (Thomine et al., 2000). OsZIP1, OsZIP3, OsZIP4, OsZIP5 and OsZIP8 are reported to encode rice plasma membrane $\mathrm{Zn}$ transporters and are induced by $\mathrm{Zn}$ deficiency (Ramesh et al., 2003; Ishimaru et al., 2005; Yang et al., 2009; Lee et al., 2010a, b and Suzuki et al., 2012). OsZIP1, OsZIP3, and OsZIP4 were expressed in the vascular bundles in shoots and in the vascular bundles and epidermal cells in roots (Ramesh et al., 2003 and Ishimaru et al., 2006).

\section{QTL for yield and its component traits for zinc deficiency tolerance in rice}

Yadav et al., 1997 used a DH population of 105 lines derived from a cross between IR64 (irrigated indica) and Azucena (upland japonica) and identified QTL regions for maximum root length (MRL) and deep root to shoot ratio (DR/SR) on chromosome 1, 2, 5, 6, 7, 8, and 9 using RFLP markers.

Avendano (2000) identified a QTL for zinc deficiency tolerance using mapping (RILs) population of Madhukar and IR26 on chromosome 5 between markers RM164 and RM87 showing a variation 61.9 per cent with a LOD value of 3.45 .

Kamoshita et al., (2008) identified QTLs using in the RILs of IR 58821/IR 52561 for root traits. They found 2, 12 and 8 QTLs for shoot biomass, deep root morphology and root thickness respectively with LOD scores ranging from 2.0 to 12.8. Phenotypic variation explained by the QTLs ranged from 6 per cent to 30 per cent. QTLs linked to seminal root length, adventitious root number, lateral root length, lateral root number and the relative parameters under flooding and upland conditions were located in RI lines developed from IR1552/Azucena (Zheng et al., 2003). A number of quantitative trait loci (QTLs) have been identified in various rice populations for various root traits including basal root thickness (Zheng et al., 2000; Price et al., 2000; Shen et al., 2001; Steele et al., 2006; Gomez et al., 2009; Kanagaraj et al., 2010; Steele et al., 2013).

Gomez et al., (2009) reported QTLs linked to physio-morphological and plant production traits under drought stress using $177 \quad \mathrm{~F}_{6}$ recombinant inbred (RI) lines of Bala $\times$ Azucena. The RI lines showed significant variation for physio-morphological and plant production traits under stress. A total of 24 QTL were identified for various traits under stress, which individually explained 4.6 to 22.3 per cent phenotypic variation. Composite interval mapping detected three markers viz., RM3894, RG409 and G1073 on chromosomes 3 and 8 linked to grain yield under drought stress, respectively explaining 22.3, 17.1 and 10.9 per cent of phenotypic variation. QTL for leaf drying, days to 50 per cent flowering and number of productive tillers under drought stress co-located at certain of these regions. Further, QTL for several root traits overlapped with QTL for grain yield under stress in these RI lines, indicating the pleiotropic effects of root trait QTL on rice performance under stress.

Thanh et al., (2006) mapped QTLs for root traits (maximum root length, root thickness, root weight to shoot and deep root weight to shoot ratios) using AFLP and SSR markers in upland rice using a recombinant inbred (RI) population derived from a cross between Vietnamese upland rice accessions. The QTL on chromosome 12 flanked by SSR marker RM270 and AFLP marker AVM28.17 and QTL on chromosome 2 flanked by markers AVM43.1-RM250were identified for maximum root length explaining phenotypic 
variance of 7.2 and 8.5 per cent respectively. For number of total tillers, the QTL on chromosome 6 flanked by markers RM50AVM29.2 were identified with the phenotypic variance of 34.70 per cent. For root weight to shoot ratio, QTL was located on chromosome 9 with phenotypic variation of 10.2 per cent flanked by RM242-RM288 markers. In addition to QTLs for root traits, QTL for plant height $(\mathrm{PH})$ on chromosome 1 flanked by markers AVM26.9-AVM26.4 with phenotypic variation of 9.70 per cent was identified.

Wissuwa et al., (2006) using a mapping population of IR64 and Jalmanga reported a QTL Zmt12 for zinc deficiency induced mortality on chromosome 12 flanked by markers CDO344-1-RG543-1 with adjusted $\mathrm{R}^{2}$ value of 11.60 and QTL Zdm3 for shoot dry matter on chromosome 3 flanked by RZ675-P1M9-10 markers with adjusted $\mathrm{R}^{2}$ value of 18.10. It was considered as a key factor for tolerance to $\mathrm{Zn}$ deficiency explaining a major portion of the variation for mortality with a LOD value of 6.40 . Stangoulis et al., (2007) detected two QTLs for zinc concentration located on chromosomes 1 and 12, explaining 15 per cent and 13 per cent of the total phenotypic variation with a LOD of 3.4 and 3.1 respectively, using a doubled haploid mapping population between IR64 and Azucena.

Garcia-Olivera et al., (2009) identified two QTLs qZN-8 and qZn-12 for Zn content using backcross populations (85 BILs) obtained by crossing Teqing (Oryza sativa) and elite wild rice (O. rufipogon) using 179 SSR markers. They found that the QTL near marker RM152 on chromosome 8 accounted for the largest proportion of phenotypic variation (11-19 per cent) for $\mathrm{Zn}$ content, whereas the QTL that was located on chromosome 12 accounted for 9 per cent phenotypic variation.
Venuprasad et al., (2009) identified two large effect QTLs DTY3.1 and DTY2.1 for grain yield under water stress in rice using RILs from the cross APO/swarna. Two markers RM234 and RM416 located on chromosome 2 and 3 respectively were shown via bulk segregant analysis to be strongly associated with yield under water stress. The QTL linked to RM416 (DTY3.1) had a large effect on yield under severe low land drought stress explaining about 31 per cent of genetic variance of the trait $(\mathrm{P}<0.0001)$. It also explained considerable variance for yield under aerobic environment. The QTL linked to RM234 (DTY2.1) had a highly significant effect on grain yield under aerobic environment explaining 16 per cent of genetic variance for the trait.

Ramya et al., (2010) concluded that the region between RM160 - RM215 on chromosome 9, contributing to maximum root depth under both control and drought condition. Primers RM242 and RM296 lying between marker interval RM160 - RM215 on chromosome 9 were reported to be linked with zinc deficiency tolerance indicating maximum root depth plays an important role in zinc deficiency tolerance mechanism.

Vikram et al., (2011) reported a major QTL qDTY1.1 for grain yield under water stress on chromosome 1 flanked by markers RM11943 and RM431 using three mapping populations. In combined analysis over two years qDTY1.1 showed an additive effect of 29.30 per cent, 24.30 per cent and 16.10 per cent of mean yield in N22 and swarna, N22 and IR64 and N22 and MTU100 respectively under water stress.

The major effect QTL for grain yield, qDTY1.1 was identified to show an effect under water stress in several genetic backgrounds. Ghimire et al., (2012) also detected qDTY1.1 in two RIL populations 
derived from donor dhagaddesi crossed to swarna and IR64 consistently over two seasons. A large effect QTL associated with grain yield in aerobic environments was identified in three genetic backgrounds. Apo/ swarna, Apo/IR72 and vandana/IR72 using bulk segregant analysis (BSA). Two closely linked rice microsatellite markers RM510 and RM19367 located on chromosome 6 were found to be associated with yield under aerobic soil conditions in all three backgrounds. The QTL linked to this marker qDTY6.1 was mapped to a $2.2 \mathrm{cM}$ region between RM19367 and RM3805 at a peak LOD score of 32 in the Apo/swarna population.

Sankar et al., (2013) reported that RM242 and RM296 primers present on chromosome 9 at locus $73.3 \mathrm{cM}$ and $20.4 \mathrm{cM}$ respectively were also found to be linked with QTL for zinc deficiency tolerance. In his study, according to root scan data obtained from field condition samples showing tolerance had more root length and root volume, which indicated that zinc deficiency tolerance character is directly or indirectly associated with the root length and root volume.

In conclusion, one of the most important uses of QTL mapping is to apply them in marker assisted selection (MAS) for genetic improvement of quantitative traits. Once the tightly linked markers have been identified, the traits can be selected indirectly using MAS. The reported map position of gramene database was used to estimate the QTLs following the inclusive composite interval mapping of additive and dominant (ICIMADD) method. The QTL analysis resulted in the identification of many QTLs for zinc deficiency tolerance in rice. Hence these QTLs may be used in Marker Assisted Selection programme (MAS) for zinc deficiency tolerance under aerobic system.

\section{References}

Avendano, B.S., 2000. Tagging high zinc content in the grain, and zinc deficiency tolerance genes in rice (Oryza sativa L.) using simple sequence repeats (SSR). M.Sc (Ag.) thesis Laguna Collage, Los Banos.

Colangelo, E.P,. and M.L. Guerinot. 2006. Put the metal to the petal: metal uptake and transport throughout plants. Curr. Opin. Plant Biol., 9: 322330.

Garcia-Olivera. A.L., T. Lubin, F. Yongcai and S. Chuanqing. 2009. QTL mapping for mineral nutrients in rice grain using introgression lines population. In: National Evaluation Central for Agricultural Wild Plant (Rice). Key Laboratory of Crop Genetic Improvement and Genome of Ministry of Agri., Beijing, China.

Ghimire, K.H., A. Q. Lenie, V. Prashant, B.P.S. Mallikarjuna, D. Shalabh, A. Helaluddin, E. H. Jose, H.B. Teresita and K. Arvind. 2012. Identification and mapping of a QTL (qdty1.1) with a consistent effect on grain yield under drought. Field Crops Res., 131: 88-96.

Gomez, M., N.M. Boopathi, S.S. Kumar, T. Ramasubramanian, Z. Chengsong, P. Jeyaprakash, A. Senthil and R.C. Babu. 2009. Molecular mapping and location of QTLs for droughtresistance traits in indica rice (Oryza sativa L.) lines adapted to target environments. Acta Physiol. Plant., 32: 355 - 364.

Ishimaru, Y., M. Suzuki, T. Kobayashi, M. Takahashi, H. Nakanishi, S. Mori and N.K. Nishizawa. 2005. OsZIP4, a novel zinc-regulated zinc transporter in rice. J. Exp. Bot., 56: 3207-3214.

Ishimaru, Y., M. Suzuki, T. Tsukamoto, K. Suzuki, M. Nakazono, T. Kobayashi, Y. Wada, S. Watanabe, S. Matsuhashi 
and M. Takahashi. 2006. Rice plants take up iron as an $\mathrm{Fe}^{3+}$. phytosiderophore and as $\mathrm{Fe}^{2+}$. Plant J., 45: 335-346.

Kamoshita, A., R.C. Babu, N.M. Boopathi and S. Fukai. 2008. Phenotypic and genotypic analysis of drought resistance traits for development of rice cultivars adapted to rainfed environments. Field Crops Res., 109: $1-23$.

Kanagaraj, P.K., S.J. Prince, J.A. Sheeba, K.R. Biji, S.B. Paul, A. Senthil and R.C. Babu. 2010. Microsatellite markers linked to drought resistance in rice (Oryza sativa L.) Curr. Sci., 98: 6.

Lee, S., H.J. Jeong, S.A. Kim, J. Lee, M.L. Guerinot and G. An. 2010b. Oszip5 is a plasma membrane zinc transporter in rice. Plant Mol. Biol., 73: 507-517.

Lee, S., S.A. Kim, J. Lee, M.L. Guerinot and G. An. 2010a. Zinc deficiencyinducible Oszip8 encodes a plasma membrane-localized zinc transporter in rice. Mol. Cells., 29: 551-558.

Morgante, M. and A.M. Olivieri. 1993. PCRamplified microsatellites as markers in plant genetics. Plant J., 3: 175-182.

Price, A.H., K.A. Steele, B.J. Moore, P.B. Barraclough and L.J. Clark. 2000. A combined RFLP and AFLP linkage map of upland rice (Oryza sativa L.) used to identify QTLs for root penetration ability. Theor. Appl. Genet., 100: 49 - 56.

Ramesh, S.A., R. Shin, D.J. Eide and D.P. Schachtman. 2003. Differential metal selectivity and gene expression of two zinc transporters from rice. Plant Physiol., 133: 126-134.

Ramya, M, M. Raveendran, Sathiyamoorthy and J. Ramalingam. 2010. In silico analysis of drought tolerant genes in rice. Int. J. Biol. Sci., 1(3): 36-40.

Sankar S.R., E., S.B. Verulkar, R.R. Saxena, S. Xalxo, S.K. Verma and P. Deepthi.
2013. Identification of qtls for tolerance to zinc deficiency in rice (Oryza sativa L). IJSID., 3(3): 317325.

Shen, L., B. Courtois, K. McNally, S. Robin and Z. Li. 2001. Evaluation of nearisogenic lines of rice introgressed with QTLs for root depth through markeraided selection. Theor. Appl. Genet., 103: 75-83.

Stangoulis, J., C.R.B. Lam, M.W. Young, C. Robin and D. Graham. 2007. Quantitative trait loci for phytate in rice grain and their relationship with grain micronutrient content. Euphytica. 154: 289-294.

Steele, K. A, A.H. Price, J.R. Witcombe, R. Shrestha, B.N. Singh, J.M. Gibbons and D.S. Virk. 2013. QTLs associated with root traits increase yield in upland rice when transferred through marker-assisted selection. Theor. Appl. Genet., 126: 101-108.

Steele, K.A., A.H. Price, H.E. Shashidhar and J.R. Witcombe. 2006. Marker-assisted selection to introgress rice QTLs controlling root traits into an Indian upland rice variety. Theor. Appl. Genet., 112: 208 - 21.

Suzuki, M., K. Bashir, H. Inoue, M. Takahashi, H. Nakanishi and N.K. Nishizawa. 2012. Accumulation of starch in Zn-deficient rice. Rice., 5: 18.

Thanh, N.D., N.T.K. Lien, P.Q. Chung, T.Q. Trong, L.T.B. Thuy and H. Nguyen. 2006. Mapping QTLs associated with root traits related to drought resistance in Vietnamese upland rice. ASEAN J. Sci. Tech. Devel., 23(4): 323-332.

Thomine, S., R. Wang, J.M. Ward, N.M. Crawford and J.I. Schroeder. 2000. Cadmium and iron transport by members of a plant metal transporter family in Arabidopsis with homology to nramp genes. Proc. Natl. Acad. 
Sci., 97: 4991-4996.

Venuprasad, R., M.E. Bool, C.O. Dalid, J. Bernier, A. Kumar, G.N. Atlin. 2009. Genetic loci responding to two cycles of divergent selection for grain yield under drought stress in a rice breeding population. Euphytica. 167: 261-269.

Vikram, P., B.P. Mallikarjuna Swamy, S. Dixit, H.U. Ahmed, M.T.S. Cruz, A.K. Singh and Arvind Kumar. 2011. qDTY1.1, a major QTL for rice grain yield under reproductive-stage drought stress with a consistent effect in multiple elite genetic backgrounds. Biomed. Genet., 12: 89.

Wissuwa, M., M. Abdelbagi, Ismail and S. Yanagihara. 2006. Effects of zinc deficiency on rice growth and genetic factors contributing to tolerance.
Plant. Physiol., 142: 731-741.

Yadav, R., B. Courtois, N. Huang and G. McLaren. 1997. Mapping genes controlling root morphology and root distribution on a double-haploid population of rice. Theor. Appl. Genet., 94: 619-632.

Yang, X., J. Huang, Y. Jiang and H.S. Zhang. 2009. Cloning and functional identification of two members of the ZIP (Zrt, Irt-like protein) gene family in rice (Oryza sativa L.). Mol. Biol. Rep., 36: 281-287.

Zheng, H., R.C. Babu, M.S. Pathan, L. Ali, N. Huang, B. Courtois and H.T. Nguyen. 2000. Quantitative trait loci for rootpenetration ability and root thickness in rice: comparison of genetic backgrounds. Genome. 43: 53 - 61.

\section{How to cite this article:}

Vanitha, J., K. Amudha, R. Mahendran, J. Srinivasan and Usha Kumari, R. 2019. A Review on Molecular Marker Analysis for Yield and its Component Traits under Water Stress and Zinc Deficiency Tolerance in Rice. Int.J.Curr.Microbiol.App.Sci. 8(05): 1013-1018. doi: https://doi.org/10.20546/ijcmas.2019.805.119 